\title{
Triplons, magnons, and spinons in a single quantum spin system: $\mathrm{SeCuO}_{3}$
}

\author{
Luc Testa $\odot,{ }^{1, *}$ Vinko Šurija, ${ }^{2}$ Krunoslav Prša $\odot,{ }^{1}$ Paul Steffens $\odot,{ }^{3}$ Martin Boehm, ${ }^{3}$ Philippe Bourges $\odot,{ }^{4}$ \\ Helmuth Berger, ${ }^{1}$ Bruce Normand, ${ }^{5,6,1}$ Henrik M. Rønnow, ${ }^{1, \dagger}$ and Ivica Živković ${ }^{1}$ \\ ${ }^{1}$ Institute of Physics, Ecole Polytechnique Fédérale de Lausanne (EPFL), CH-1015 Lausanne, Switzerland \\ ${ }^{2}$ Institute of Physics, Bijenička 46, HR-10000 Zagreb, Croatia \\ ${ }^{3}$ Institut Laue-Langevin, BP 156, 38042 Grenoble Cedex 9, France \\ ${ }^{4}$ Université Paris-Saclay, CNRS, CEA, Laboratoire Léon Brillouin, 91191 Gif-sur-Yvette, France \\ ${ }^{5}$ Paul Scherrer Institute, $\mathrm{CH}-5232$ Villigen PSI, Switzerland \\ ${ }^{6}$ Lehrstuhl für Theoretische Physik I, Technische Universität Dortmund, Otto-Hahn-Strasse 4, 44221 Dortmund, Germany
}

(Received 3 July 2020; revised 17 December 2020; accepted 6 January 2021; published 25 January 2021; corrected 1 February 2021)

\begin{abstract}
Quantum magnets display a wide variety of collective excitations, including spin waves (magnons), coherent singlet-triplet excitations (triplons), and pairs of fractional spins (spinons). These modes differ radically in nature and properties, and in all conventional analyses any given material is interpreted in terms of only one type. We report inelastic neutron scattering measurements on the spin-1/2 antiferromagnet $\mathrm{SeCuO}_{3}$, which demonstrate that this compound exhibits all three primary types of spin excitation. $\mathrm{Cu}_{1}$ sites form strongly bound dimers while $\mathrm{Cu}_{2}$ sites form a network of spin chains, whose weak three-dimensional (3D) coupling induces antiferromagnetic order. We perform quantitative modeling to extract all of the relevant magnetic interactions and show that magnons of the $\mathrm{Cu}_{2}$ system give a lower bound to the spinon continua, while the $\mathrm{Cu}_{1}$ system hosts a band of high-energy triplons at the same time as frustrating the 3D network.
\end{abstract}

DOI: 10.1103/PhysRevB.103.L020409

The exotic collective excitations observed in magnetic materials emerge from the rich spectrum of possible effects when quantum spin fluctuations act in different geometries, dimensionalities, and with different degrees of frustration. When fluctuations push a system beyond robust magnetic order and textbook spin waves, common types of excitation include triplons arising from structural dimerization [1-3] and frustration [4], bound states of magnons and triplons [5-8], and fractions of magnons and triplons; these latter include spinons [2,9,10], solitons [11,12], Majorana quasiparticles [13-15], and other topological objects. In all these situations, the system is normally analyzed in terms of just one type of excitation, and detailed theoretical and numerical approaches have been developed for comparison with experiment.

However, an often overlooked category is the set of quantum magnets in which magnetic order is present only as a rather thin veneer on a "background" dominated by quantum fluctuations. While the Bragg peaks and magnons of the ordered spin component tend to dominate the measured experimental response, no rule states that the remaining spin fluctuations should be incoherent. The field- [16] and pressure-induced [17] quantum phase transitions of $\mathrm{TlCuCl}_{3}$ provide an example of arbitrarily weak antiferromagnetic order superposed on a fluctuating dimer system with triplon excitations. In $\mathrm{KCuF}_{3}$, weak coupling of the spin chains produces magnetic order superposed on a system of spinons,

\footnotetext{
*luc.testa@gmail.com

†henrik.ronnow@epfl.ch
}

which is revealed at high energy [18]. Candidate spinon excitations also coexist with square-lattice antiferromagnetism [19], and many low-dimensional metal-organic systems offer the possibility of controlling this coexistence [20]. One structural route to the same phenomena is provided by the spin-tetrahedron material $\mathrm{Cu}_{2} \mathrm{Te}_{2} \mathrm{O}_{5} X_{2}(X=\mathrm{Cl}, \mathrm{Br})$ [21], where the magnetic response is dominated by the nonordered spins [22], a situation anticipated in theory [23], and incommensurate magnetism [24] coexists with anomalous coupled-cluster excitations [25]; similar coupled-cluster physics has been pursued in a number of other materials [26-28]. Another is the recent discovery [29] of both spinons and magnons in very weakly coupled chains of two different types.

Here we investigate the coexistence of multiple excitation types by an inelastic neutron scattering (INS) study of $\mathrm{SeCuO}_{3}$. This compound displays both quasilocalized highenergy states and weak magnetic order at low temperatures. We demonstrate that the excitation spectrum has one triplon branch, dispersing weakly around $27 \mathrm{meV}$, and a magnonlike branch below $4 \mathrm{meV}$, whose associated scattering intensity shows the clear fingerprints of spinon continua. By model calculations using linear spin-wave theory and perturbative methods, we deduce the interaction parameters of a minimal magnetic Hamiltonian, allowing us to describe $\mathrm{SeCuO}_{3}$ in terms of two interacting spin subsystems, namely, dimers and chains, each of which shapes the magnetic excitations of the other.

The $S=1 / 2$ quantum magnet $\mathrm{SeCuO}_{3}$ [30] has a monoclinic unit cell with space group $P 2_{1} / n$ and lattice parameters $a=7.71 \AA, b=8.24 \AA, c=8.50 \AA$, and $\beta=99.12^{\circ}$. Two 


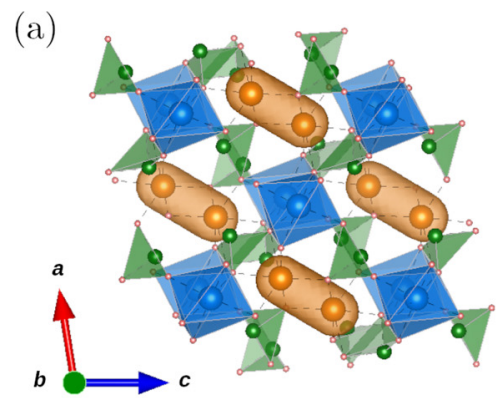

(c)

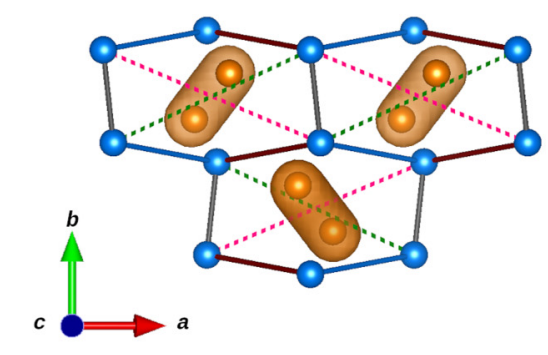

(d) (e)
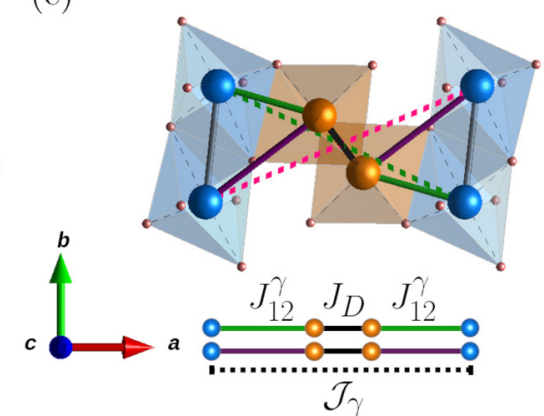

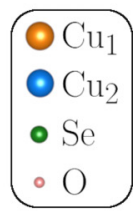

(b)

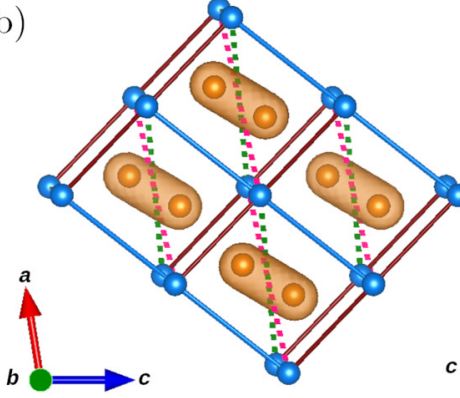

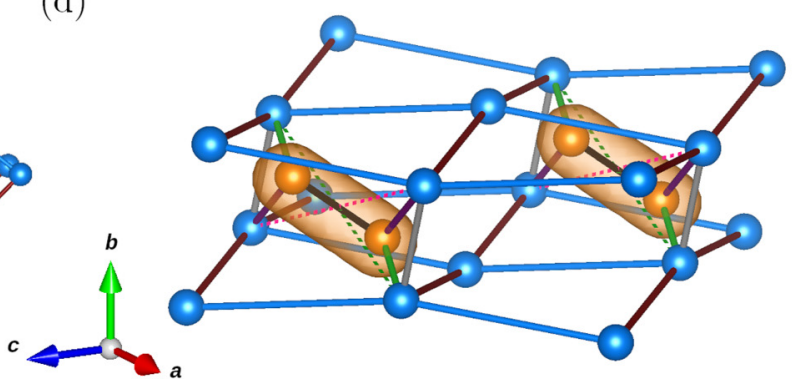

FIG. 1. Atomic structure and magnetic interactions of $\mathrm{SeCuO}_{3}$. (a) Schematic representation of the atomic structure showing $\mathrm{Cu}_{1}$ (orange), $\mathrm{Cu}_{2}$ (blue), Se (green), and $\mathrm{O}$ (pink) atoms. (b) Projection on the $a c$ and (c) on the $a b$ plane, indicating the magnetic interactions of Table I. (d) Perspective view highlighting the $\mathrm{Cu}_{2}$ chains and the direct interactions connecting them into coupled, buckled planes. (e) Geometry of the effective interactions mediated between $\mathrm{Cu}_{2}$ atoms by the $\mathrm{Cu}_{1}$ dimer units: $\mathcal{J}_{\alpha}$ and $\mathcal{J}_{\beta}$ are given in terms of the two different $\mathrm{Cu}_{1}-\mathrm{Cu}_{2}$ interactions, $J_{12}^{\alpha}$ (green) and $J_{12}^{\beta}$ (purple), and the dimer interaction, $J_{D}$ (black), by Eq. (2).

crystallographically inequivalent $\mathrm{Cu}$ sites, $\mathrm{Cu}_{1}$ and $\mathrm{Cu}_{2}$, are each surrounded by six $\mathrm{O}$ atoms, forming $\mathrm{CuO}_{4}$ plaquettes, with the remaining two $\mathrm{O}$ atoms forming the elongated octahedra represented for the $\mathrm{Cu}_{2}$ atoms in Figs. 1(a) and 1(e). This elongation favors the $d_{x^{2}-y^{2}}$ orbitals, ensuring strong $\mathrm{Cu}_{1}$ dimer units (orange shading in Fig. 1) of edge-sharing plaquettes whose superexchange paths have a $\mathrm{Cu}_{1}-\mathrm{O}-\mathrm{Cu}_{1}$ angle of $101.9^{\circ}$ [31]. Including the $\mathrm{Cu}_{2} d_{x^{2}-y^{2}}$ orbitals led to the proposal of a weakly coupled network of linear $\left(\mathrm{Cu}_{2}-\mathrm{Cu}_{1}-\mathrm{Cu}_{1}-\mathrm{Cu}_{2}\right)$ tetramers [31], although this scenario cannot explain the magnetic susceptibility below $90 \mathrm{~K}$. Recent nuclear quadrupole resonance measurements confirmed the formation of singlet states at $T \lesssim 200 \mathrm{~K}$ [32], and together with nuclear magnetic resonance, electron spin resonance, and torque magnetometry experiments $[33,34]$ were interpreted as reflecting two essentially decoupled subsystems, the strong, local $\mathrm{Cu}_{1}$ dimers and weakly coupled $\mathrm{Cu}_{2}$ spins hosting magnetic order below $T_{N}=8 \mathrm{~K}$.

To access the full spin dynamics of $\mathrm{SeCuO}_{3}$, we grew a $1 \mathrm{~g}$ single-crystal sample by chemical vapor transport. Thermal neutron INS measurements were performed on the IN8 spectrometer (ILL [35]) to probe the $(h k h)$ scattering plane. The low-energy dynamics were studied on the cold-neutron spectrometers ThALES (ILL [36]) and 4F1 (LLB), the latter experiment probing the $(h k \bar{h})$ scattering plane. Full details of the instrumental setups employed are provided in Sec. S1 of the Supplemental Material (SM) [37]. The measured intensities, $I(\mathbf{q}, \omega)$, are directly proportional to the dynamical structure factor, $S(\mathbf{q}, \omega)$, for scattering processes at momentum transfer $\mathbf{q}$ and energy transfer $\omega$.

In Fig. 2 we present the high-energy dynamics of $\mathrm{SeCuO}_{3}$ as measured on IN8. We obtained $I(\mathbf{q}, \omega)$ for $\mathbf{q}$ points along two orthogonal high-symmetry directions. At $2 \mathrm{~K}$, each energy scan [Fig. 2(a)] has a resolution-limited peak that we fit with a Gaussian at all $\mathbf{q}$ points to extract a weak dispersion around $26.5 \mathrm{meV}$ [Fig. 2(b)], with smooth changes in intensity [Fig. 2(c)]. At $15 \mathrm{~K}$, i.e., above $T_{N}$, the peak shows only a minimal downward shift and increased broadening [Fig. 2(a)]. Figures 2(d)-2(f) confirm that this mode persists at least until $120 \mathrm{~K}$, i.e., far beyond $T_{N}$, and that its width is captured by the Lorentzian component of a Voigt line shape.

The weak $\mathbf{q}$ dependence of this excitation indicates its nature as a near-localized triplon of the $\mathrm{Cu}_{1}$ dimers, whose energy is given by $J_{D}$ in Fig. 1. Its Lorentzian width increases linearly with temperature until a value of $4 \mathrm{meV}$ [Figs. 2(d) and 2(e)], which we will show reflects the coupling to the excitations of the $\mathrm{Cu}_{2}$ subsystem. However, the primary thermal effect is intrinsic, as shown in Fig. 2(f) by comparing the mode amplitude with the probability, $\left[1+3 \exp \left(-J_{D} / k_{\mathrm{B}} T\right)\right]^{-1}$, of finding a $J_{D}$ dimer in its singlet state at temperature $T$, which further confirms the triplon nature of this mode.

Turning to the low-energy dynamics measured at $2 \mathrm{~K}$ on ThALES and 4F1, representative background-subtracted constant-q $\omega$ scans are shown in Fig. 3. A strong low-energy mode is present at all $\mathbf{q}$, but a continuum of scattering states persists above this feature, at least up to the highest measured energy of $4.5 \mathrm{meV}$. To visualize this continuum scattering, we present our intensity data as color maps in Fig. 4, and note that it appears in all three dimensions of reciprocal space. We return below to a detailed discussion of this continuum.

For a systematic analysis we perform a Gaussian fit to the peak at the lower edge of the continuum (Fig. 3) and collect two separate intensity contributions, $I_{\mathrm{p}}$ from the Gaussian and $I_{\mathrm{c}}$ from the excess scattering at all higher energies. The upper panels of Fig. 4 show the values of $I_{\mathrm{p}}(\mathbf{q})$ and $I_{\mathrm{c}}(\mathbf{q})$ extracted from 74 energy scans. The lower panels show a 


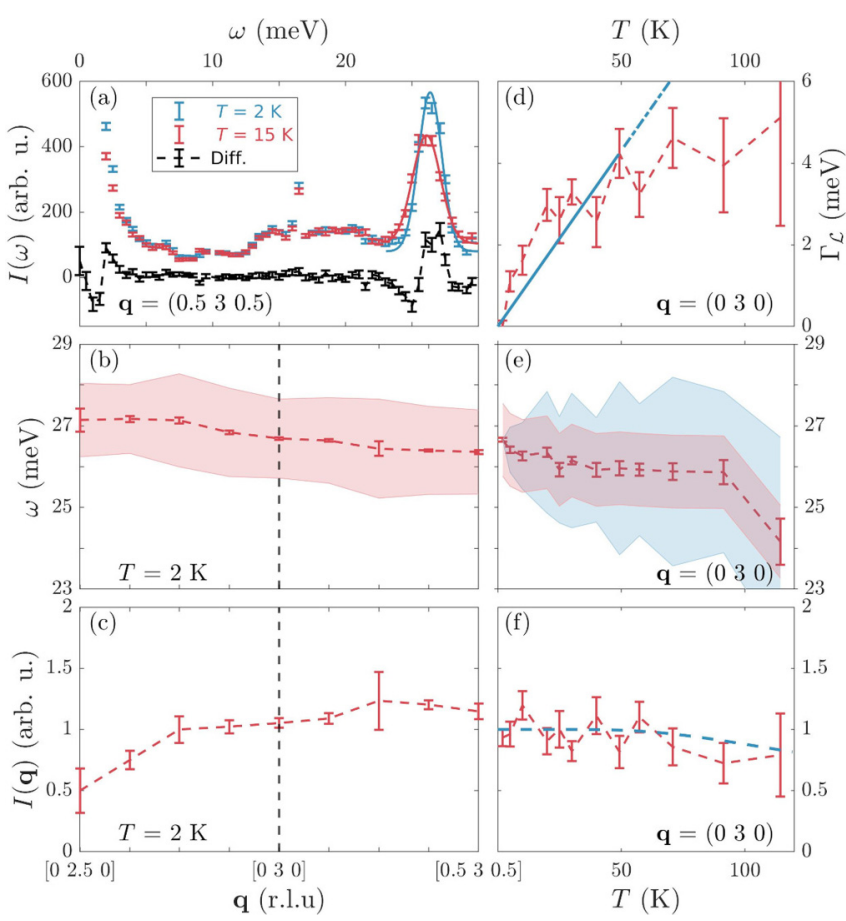

FIG. 2. Triplon excitation. (a) Intensity, $I(\omega)$, at $\mathbf{q}=\left(\begin{array}{lll}0.5 & 3 & 0.5\end{array}\right)$, measured at low (blue) and intermediate (red) temperatures with the difference shown in black. (b) Dispersion, $\omega(\mathbf{q})$, of the triplon in two orthogonal q directions; shading represents the width (FWHM) of the mode at each $\mathbf{q}$ point. (c) Integrated intensity, $I(\mathbf{q})$, for the same directions. (d)-(f) Thermal evolution at $\mathbf{q}=\left(\begin{array}{lll}0 & 3 & 0\end{array}\right)$. (d) Lorentzian width, $\Gamma_{\mathcal{L}}$ (red), compared to $k_{\mathrm{B}} T$ (blue). (e) $\omega(\mathbf{q}, T)$; shading indicates the instrumental resolution of $1.8(2) \mathrm{meV}$ (red) and the Lorentzian profile (blue). (f) Normalized $I(\mathbf{q})$ in red compared with the thermal singlet population (blue).

well-defined band with a maximum of $4 \mathrm{meV}$ and a small gap, $\Delta=0.42(3) \mathrm{meV}$, where $I_{\mathrm{p}}(\mathbf{q})$ becomes large due to the magnetic order.

We expect that, with the exception of a term opening the gap, the minimal magnetic Hamiltonian contains only Heisenberg interactions between near-neighbor spins in all directions, and thus takes the form

$$
\hat{\mathcal{H}}=J_{D} \sum_{\left\langle i_{1}, j_{1}\right\rangle} \hat{\mathbf{S}}_{i_{1}} \cdot \hat{\mathbf{S}}_{j_{1}}+\sum_{\left\langle i_{1}, i_{2}\right\rangle, \gamma} J_{12}^{\gamma} \hat{\mathbf{S}}_{i_{1}} \cdot \hat{\mathbf{S}}_{i_{2}}+\sum_{\left[i_{2}, j_{2}\right]_{m}} J_{m} \hat{\mathbf{S}}_{i_{2}} \cdot \hat{\mathbf{S}}_{j_{2}} .
$$

Here $i_{1}$ and $j_{1}$ denote $\mathrm{Cu}_{1}$ sites and $i_{2}$ and $j_{2} \mathrm{Cu}_{2}$ sites, $\langle\cdots\rangle$ denotes a sum restricted to nearest-neighbor bonds, and $[\cdots]_{m}$ a sum over bonds in the set $\left\{J_{m}\right\}$. Having interpreted the high-energy response as a triplon of the $\mathrm{Cu}_{1}$ subsystem with $J_{D}=26.5 \mathrm{meV}$, we build up our knowledge of the terms in Eq. (1) by next describing the low-energy response as a consequence of the decoupled $\mathrm{Cu}_{2}$ subsystem, i.e., by neglecting the second term. For this we seek a set of interaction parameters that, used in an effective Hamiltonian of the same form as the last term of Eq. (1), reproduces the magnon dispersions and intensities in Fig. 4. As shown in Fig. 1, we allow both near-neighbor couplings, $\left\{J_{m}\right\}$, and long-distance "effective" couplings, $\left\{\mathcal{J}_{\gamma}\right\}$, over paths that include the polyhedra of other $\mathrm{Cu}_{1}$ atoms and whose microscopic origin therefore lies in the second term of Eq. (1), as we discuss further below.

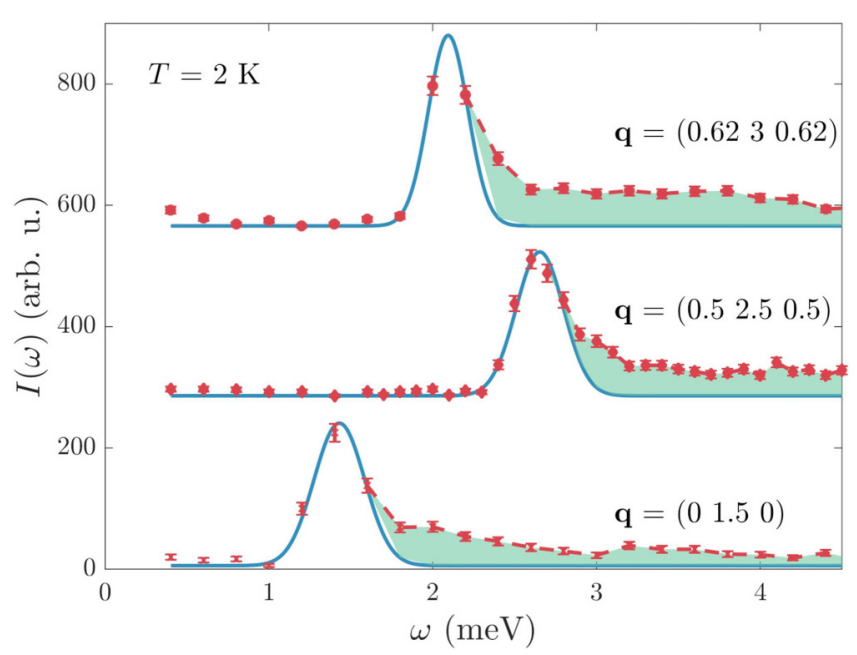

FIG. 3. Magnon peak and scattering continua. $I(\omega)$ at three different $\mathbf{q}$ points. Measured data (red points) are fitted by a Gaussian peak (blue line) at the lower edge. Green shading indicates a scattering continuum at higher energies.

We fit $\omega(\mathbf{q})$ using linear spin-wave (LSW) theory, as implemented in the SPINW package [38], obtaining an excellent account of the INS peak positions when the interaction parameters of Fig. 1 have the values reported in Table I. This fit contains two magnon branches, one of which has over $90 \%$ of the intensity we measure. The LSW theory delivers an accurate account of $I_{\mathrm{p}}(\mathbf{q})$ for the strong branch with no further fitting, as Fig. 4 makes clear, underlining its success in capturing all the leading physics of the magnon spectrum. Turning to percent-level discrepancies, in the LSW treatment the weak branch has a very low $[\mathcal{O}(0.1 \%)]$ intensity, whereas the intensities measured in Fig. 4 are in general 1\%-5\% of the strong branch. While this discrepancy suggests that the magnetic Hamiltonian of $\mathrm{SeCuO}_{3}$ contains further terms (beyond those in Table I) coupling two quasiindependent magnetic sublattices, the low intensity indicates that they are very weak.

The interactions of Table I define a $\mathrm{Cu}_{2}$ magnetic lattice composed of chains aligned in the $\mathbf{a}-\mathbf{c}$ direction, whose energy scale, $J_{\|}$, exceeds by a factor of 10 all the interchain couplings. From Fig. $1(\mathrm{a}), J_{\|}$connects $\mathrm{Cu}_{2}$ spins through the $\mathrm{SeO}_{3}$ tetrahedra, a superexchange path not so far considered [31-34]. This coupled-chain character provides an immediate indication for the origin of the continuum scattering in Figs. 3 and 4 as the break-up of $\Delta S=1$ magnons into spinons at energies beyond their confinement scale. The four additional $\mathrm{Cu}_{2}$ interactions ensure both strong interchain frustration in all three directions and the weak magnetic order at $T<T_{N}$.

TABLE I. Superexchange interaction parameters within the $\mathrm{Cu}_{1}$ and $\mathrm{Cu}_{2}$ subsystems, in $\mathrm{meV}$, obtained by fitting the dispersion data of Figs. 2(b) and 4. The geometry of the interactions is shown in Fig. 1.

\begin{tabular}{lccccc}
\hline \hline $\mathrm{Cu}_{1}-\mathrm{Cu}_{1}$ & & \multicolumn{5}{c}{$\mathrm{Cu}_{2}-\mathrm{Cu}_{2}$} \\
\hline$J_{D}$ & $J_{\|}$ & $J_{\perp}$ & $J_{b}$ & $\mathcal{J}_{\alpha}$ & $\mathcal{J}_{\beta}$ \\
$26.5(6)$ & $3.39(13)$ & $0.39(3)$ & $-0.19(2)$ & $0.34(2)$ & $0.35(2)$ \\
\hline \hline
\end{tabular}




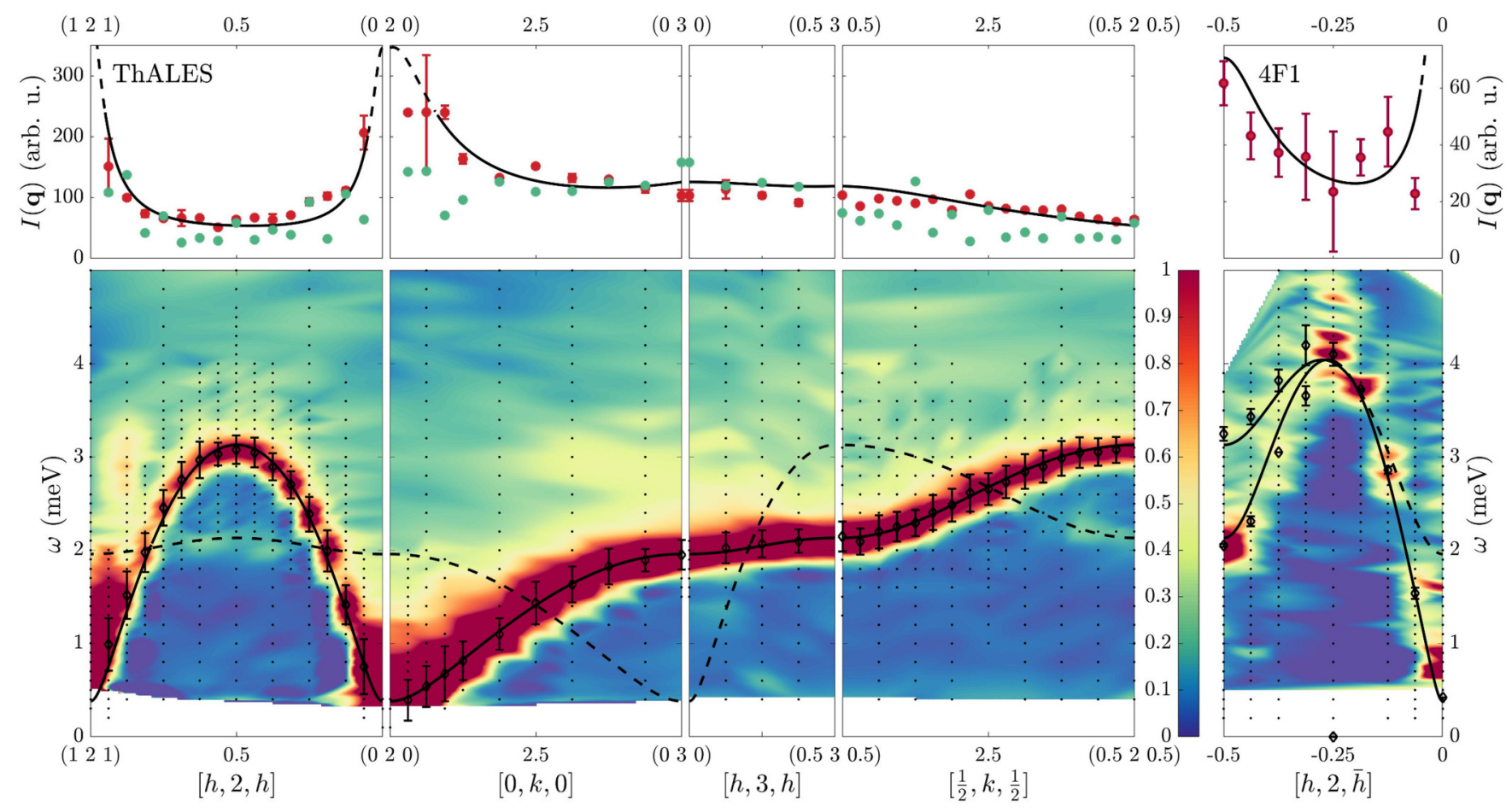

FIG. 4. Magnon and spinon spectra. Colored panels show the scattering intensity, $S(\mathbf{q}, \omega)$, for five different $\mathbf{q}$ directions. The black points show the peak centers taken from Fig. 3 and the error bars the extracted width (FWHM) of the Gaussian profiles. The black lines show the two spin-wave branches in the model fit to the lower peak of Fig. 3, one of which (dashed line) has vanishing intensity. The upper panels show the integrated intensities, $I(\mathbf{q})$, of the measured spin-wave contribution $\left[I_{\mathrm{p}}(\mathbf{q})\right.$, red points $]$ and the continuum contribution $\left[I_{\mathrm{c}}(\mathbf{q})\right.$, green points $]$, taken respectively from the peak and shaded areas in Fig. 3; the black lines show the spin-wave intensities given by the model parameters. In the panel at right, the intensity is a sum of two modes.

The small magnon gap can be reproduced with a tiny Ising anisotropy of $\boldsymbol{J}_{\|}, \delta J_{\|}=0.018 \mathrm{meV}$, which has negligible influence on the dynamics away from the zone center.

To go beyond the independent-subsystem interpretation of the excitation spectrum, we restore the coupling between $\mathrm{Cu}_{1}$ and $\mathrm{Cu}_{2}$ atoms in the second term of Eq. (1), without which the triplon measured in Fig. 2 would be nondispersive. All the interactions between the $\mathrm{Cu}_{2}$ atoms given in Table I are required to fit the dispersion data of Fig. 4 in multiple reciprocal-space directions. However, they include not only the near-neighbor couplings $J_{\|}, J_{\perp}$, and $J_{b}$, but also the couplings $\mathcal{J}_{\alpha}$ and $\mathcal{J}_{\beta}$ whose long superexchange paths proceed directly across the $\mathrm{Cu}_{1}-\mathrm{Cu}_{1}$ dimer (Fig. 1); as a result, in a self-consistent model, these should be effective couplings arising as a consequence of $J_{D}$ and of two $\mathrm{Cu}_{1}-\mathrm{Cu}_{2}$ coupling parameters, $J_{12}^{\gamma}$ [Fig. 1(e)].

To estimate $J_{12}^{\gamma}$, we perform a perturbative analysis of the two four-site units shown in Fig. 1(e), as detailed in Sec. S2 of the SM [37]. The ground state in the limit $J_{D} \gg J_{12}^{\gamma},\left|\Phi_{0}\right\rangle=$ $\left|s_{1}\right\rangle \otimes\left|s_{2}\right\rangle$, is the product of two singlets on each pair of $\mathrm{Cu}_{1}$ and $\mathrm{Cu}_{2}$ sites. In the three lowest excited states, the $\mathrm{Cu}_{1}$ dimer remains in a singlet while the $\mathrm{Cu}_{2}$ spins form a triplet, $\left|t_{2}^{l}\right\rangle$, with $l=+, 0,-$. The energy difference gives the effective coupling between the two $\mathrm{Cu}_{2}$ atoms,

$$
\mathcal{J}_{\gamma}=\frac{J_{12}^{\gamma}}{2}+\frac{1}{4} \frac{\left[3\left(J_{12}^{\gamma}\right)^{2}-2 J_{D} J_{12}^{\gamma}\right]}{\sqrt{J_{D}^{2}+\left(J_{12}^{\gamma}\right)^{2}}} \stackrel{J_{D} \gg J_{12}^{\gamma}}{\longrightarrow} \frac{3}{4} \frac{\left(J_{12}^{\gamma}\right)^{2}}{J_{D}} .
$$

From the fitted values of the effective couplings $\mathcal{J}_{\alpha}$ and $\mathcal{J}_{\beta}$ (Table I), we deduce the microscopic coupling parameters to be $J_{12}^{\alpha}=3.47 \mathrm{meV}$ and $J_{12}^{\beta}=3.52 \mathrm{meV}$. Our LSW fits verify that any effective coupling between atoms connected by a path involving both $J_{12}^{\alpha}$ and $J_{12}^{\beta}$ must be vanishingly small.

Although these values are large compared to the couplings within the $\mathrm{Cu}_{2}$ subsystem in Table I, their real effect on the spin dynamics is suppressed strongly by $J_{D}$, as the structure of Eq. (2) makes clear. Values of 3-4 meV are consistent with the width, $\Gamma_{\mathcal{L}}$, of the triplon at high temperatures [Fig. 2(d)], which indicates its coupling to incoherent excitations. The perturbative treatment of Eq. (2) provides upper bounds for the $J_{12}^{\gamma}$ values and thus is the opposite limit to the LSW approach, which cannot describe the full system of $\mathrm{Cu}_{1}$ and $\mathrm{Cu}_{2}$ atoms. The two approaches indicate the range of possible renormalization effects due to quantum fluctuations, which is widest at intermediate energies (corresponding to the spinon continua). The extent of renormalization to LSW theory in $\mathrm{SeCuO}_{3}$ can be gauged from the magnetic order on the $\mathrm{Cu}_{2}$ sublattice, which despite its three-dimensional (3D) nature is $\mu_{2}<0.8 \mu_{B}$ [32]. The interactions $J_{12}^{\gamma}$ induce order on the $\mathrm{Cu}_{1}$ sublattice, although $\mu_{1} \approx 0.35 \mu_{B}$ is very weak even at the lowest temperatures, and hence a full description lies well beyond the LSW approximation. The relative canting of the $\mu_{1}$ and $\mu_{2}$ moment directions [31] and the intensity transfer to the weak magnon branch (Fig. 4) gauge the physical effects of terms omitted in the minimal model of Eq. (1). 
TABLE II. Integrated peak intensity, $I_{\mathrm{p}}$, and continuum intensity, $I_{\mathrm{c}}$, averaged along four high-symmetry directions. The lower row presents the LSW theoretical result for the spin reduction, $\Delta S_{2}=0.13$, measured [32] on the $\mathrm{Cu}_{2}$ sublattice.

\begin{tabular}{lrrc}
\hline \hline Direction & \multicolumn{1}{c}{$I_{\mathrm{p}}$} & \multicolumn{1}{c}{$I_{\mathrm{c}}$} & $\kappa_{\min }$ \\
\hline$[h, 2, h]$ & $90(4)$ & 61 & $0.69(4)$ \\
{$[0, k, 0]$} & $133(5)$ & 99 & $0.85(4)$ \\
{$[h, 3, h]$} & $98(4)$ & 125 & $1.28(4)$ \\
{$\left[\frac{1}{2}, k, \frac{1}{2}\right]$} & $85(4)$ & 56 & $0.65(4)$ \\
$\Delta S_{2}=0.13$ & 0.47 & 0.15 & 0.32 \\
\hline \hline
\end{tabular}

We return now to the most unexpected feature of our INS data, the strong continuum scattering observed directly above the magnon peaks in Figs. 3 and 4. Interpreting this as deconfined spinons requires the definitive exclusion of alternative origins. Continuum scattering above a one-magnon band arises naturally due to multimagnon processes and has long been known in both 3D [39] and 2D antiferromagnets [40]. In this situation, the ratio of the integrated intensities, $I_{\mathrm{p}}$ in the one-magnon sector and $I_{\mathrm{c}}$ in the putative multimagnon sector (Fig. 4), cannot exceed a well-defined limit. In Table II we average both quantities along four $\mathbf{q}$ directions, and note further that our measured energy range may not capture the upper edge of the continuum (Fig. 3), whence the ratio $\kappa_{\min }=I_{\mathrm{c}} / I_{\mathrm{p}}$ constitutes a lower bound.

We find that $I_{\mathrm{c}}$ is of the same order as $I_{\mathrm{p}}$, making their ratio far greater than those found in multimagnon scattering studies $[39,40]$. The LSW prediction for this ratio can be deduced from the spin reduction (quantum fluctuation renormalization) measured [32] on the $\mathrm{Cu}_{2}$ sublattice, and as Table II makes clear the data exclude any straightforward multimagnon origin. We comment that highly unconventional multimagnon processes, such as those involving very strong magnon-magnon interactions, are hard to rule out, but that there is little evidence for these (such as decay of our observed magnons) in $\mathrm{SeCuO}_{3}$. Further, the gap in the one-magnon spectrum implies a gap between the one- and multimagnon contributions, as encountered in Ref. [40], whereas Figs. 3 and 4 exclude such a gap with even a fraction the size of the measured $\Delta$.

Thus we conclude that the most plausible origin for the observed continuum scattering is spinons. More specifically, the strong quantum corrections of the chainlike $S=1 / 2 \mathrm{Cu}_{2}$ system do permit a deconfinement of spinons at energies above the one-magnon band. However, the frustrating interchain interactions, which allow this spinonic character in a system with magnetic order, mean that the resulting continua (Fig. 4) are far from the familiar single-chain form, which was found in Ref. [29] for magnons and spinons coexisting in the quasi-1D limit. The problem of partially confined spinons has recently received considerable attention in some of the paradigm Heisenberg models within frustrated quantum magnetism [41-45], and $\mathrm{SeCuO}_{3}$ presents a materials example of this complex situation. While a detailed analysis lies beyond the scope of the present study, the locations of continuum scattering in Fig. 4 and the intensity ratios for magnon and spinon contributions will provide essential input for a complete theoretical description.

To conclude, we have investigated a member of the class of coupled-cluster, multisubsystem quantum magnetic materials in which magnon, triplon, and spinon excitations are present simultaneously. In $\mathrm{SeCuO}_{3}$, the clusters are strong $\mathrm{Cu}_{1}$ dimers and the second sublattice, $\mathrm{Cu}_{2}$, is a network of spin chains on which weak magnetic order appears below $T_{N}=8 \mathrm{~K}$. By neutron spectroscopy we have determined not only the intrasublattice interactions but also the $\mathrm{Cu}_{1}-\mathrm{Cu}_{2}$ interactions that make the $\mathrm{Cu}_{1}$ triplon mode weakly dispersive, induce small $\mathrm{Cu}_{1}$ moments, and create frustrating interactions in the $\mathrm{Cu}_{2}$ sublattice, which contribute to the emergence of spinon continua above the magnons. From our results, $\mathrm{SeCuO}_{3}$ encapsulates the challenge of describing the coherent quantum correlations, or quantum entanglement, that in many systems lie beneath the (unentangled) veneer of magnetic order, and mandates an integrated theoretical treatment of how these correlations lead to all three coexisting excitation types.

We thank X. Rocquefelte for helpful discussions. We are grateful to the Swiss National Science Foundation (SNSF) for financial support under Grant No. 188648 and the European Research Council (ERC) for the support of the Synergy Grant HERO. Research at TU Dortmund was supported by the Deutsche Forschungsgemeinschaft (DFG, German Research Foundation) through Grants No. UH90/13-1 and No. UH90/14-1. We thank the Institut Laue-Langevin and the Laboratoire Léon Brillouin for access to their neutron scattering facilities. These neutron scattering experiments were performed within the framework of the EUROfusion Consortium, which was funded by the Euratom Research and Training Programme 2014-2018 under Grant Agreement No. 633053.
[1] N. Cavadini, G. Heigold, W. Henggeler, A. Furrer, H.-U. Güdel, K. Krämer, and H. Mutka, Phys. Rev. B 63, 172414 (2001).

[2] B. Thielemann, C. Rüegg, H. M. Rønnow, A. M. Läuchli, J.-S. Caux, B. Normand, D. Biner, K. W. Krämer, H.-U. Güdel, J. Stahn, K. Habicht, K. Kiefer, M. Boehm, D. F. McMorrow, and J. Mesot, Phys. Rev. Lett. 102, 107204 (2009).

[3] D. Schmidiger, S. Mühlbauer, A. Zheludev, P. Bouillot, T. Giamarchi, C. Kollath, G. Ehlers, and A. M. Tsvelik, Phys. Rev. B 88, 094411 (2013).
[4] B. D. Gaulin, S. H. Lee, S. Haravifard, J. P. Castellan, A. J. Berlinsky, H. A. Dabkowska, Y. Qiu, and J. R. D. Copley, Phys. Rev. Lett. 93, 267202 (2004).

[5] J. B. Torrance and M. Tinkham, Phys. Rev. 187, 595 (1969).

[6] G. S. Uhrig and H. J. Schulz, Phys. Rev. B 54, R9624 (1996).

[7] S. Ward, M. Mena, P. Bouillot, C. Kollath, T. Giamarchi, K. P. Schmidt, B. Normand, K. W. Krämer, D. Biner, R. Bewley, T. Guidi, M. Boehm, D. F. McMorrow, and C. Rüegg, Phys. Rev. Lett. 118, 177202 (2017). 
[8] P. A. McClarty, F. Krüger, T. Guidi, S. F. Parker, K. Refson, A. W. Parker, D. Prabhakaran, and R. Coldea, Nat. Phys. 13, 736 (2017).

[9] P. R. Hammar, M. B. Stone, D. H. Reich, C. Broholm, P. J. Gibson, M. M. Turnbull, C. P. Landee, and M. Oshikawa, Phys. Rev. B 59, 1008 (1999).

[10] M. Mourigal, M. Enderle, A. Klöpperpieper, J.-S. Caux, A. Stunault, and H. M. Rønnow, Nat. Phys. 9, 435 (2013).

[11] S. E. Nagler, W. J. L. Buyers, R. L. Armstrong, and B. Briat, Phys. Rev. B 28, 3873 (1983).

[12] Q. Faure, S. Takayoshi, S. Petit, V. Simonet, S. Raymond, L.-P. Regnault, M. Boehm, J. S. White, M. Månsson, C. Rüegg, P. Lejay, B. Canals, T. Lorenz, S. C. Furuya, T. Giamarchi, and B. Grenier, Nat. Phys. 14, 716 (2018).

[13] A. Kitaev, Ann. Phys. 321, 2 (2006).

[14] J. Chaloupka, G. Jackeli, and G. Khaliullin, Phys. Rev. Lett. 105, 027204 (2010).

[15] J. Wang, B. Normand, and Z.-X. Liu, Phys. Rev. Lett. 123, 197201 (2019).

[16] C. Rüegg, N. Cavadini, A. Furrer, H.-U. Güdel, K. Krämer, H. Mutka, A. Wildes, K. Habicht, and P. Vorderwisch, Nature (London) 423, 62 (2003).

[17] C. Rüegg, B. Normand, M. Matsumoto, A. Furrer, D. F. McMorrow, K. W. Krämer, H. U. Güdel, S. N. Gvasaliya, H. Mutka, and M. Boehm, Phys. Rev. Lett. 100, 205701 (2008).

[18] B. Lake, D. A. Tennant, C. D. Frost, and S. E. Nagler, Nat. Mater. 4, 329 (2005).

[19] B. Dalla Piazza, M. Mourigal, N. B. Christensen, G. J. Nilsen, P. Tregenna-Piggott, T. G. Perring, M. Enderle, D. F. McMorrow, D. A. Ivanov, and H. M. Rønnow, Nat. Phys. 11, 62 (2015).

[20] M. Skoulatos, M. Månsson, C. Fiolka, K. W. Krämer, J. Schefer, J. S. White, and C. Rüegg, Phys. Rev. B 96, 020414(R) (2017).

[21] M. Johnsson, K. W. Törnroos, F. Mila, and P. Millet, Chem. Mater. 12, 2853 (2000).

[22] Z. Jagličić, S. El Shawish, A. Jeromen, A. Bilušić, A. Smontara, Z. Trontelj, J. Bonča, J. Dolinšek, and H. Berger, Phys. Rev. B 73, 214408 (2006).

[23] V. N. Kotov, M. E. Zhitomirsky, M. Elhajal, and F. Mila, Phys. Rev. B 70, 214401 (2004).

[24] O. Zaharko, A. Daoud-Aladine, S. Streule, J. Mesot, P.-J. Brown, and H. Berger, Phys. Rev. Lett. 93, 217206 (2004).

[25] K. Prša, H. M. Rønnow, O. Zaharko, N. B. Christensen, J. Jensen, J. Chang, S. Streule, M. Jiménez-Ruiz, H. Berger, M. Prester, and J. Mesot, Phys. Rev. Lett. 102, 177202 (2009).

[26] R. Becker, M. Johnsson, R. K. Kremer, and P. Lemmens, J. Solid State Chem. 178, 2024 (2005).
[27] O. Zaharko, J. Mesot, L. A. Salguero, R. Valentí, M. Zbiri, M. Johnson, Y. Filinchuk, B. Klemke, K. Kiefer, M. Mys'kiv, T. Strässle, and H. Mutka, Phys. Rev. B 77, 224408 (2008).

[28] K.-Y. Choi, S. Do, P. Lemmens, J. van Tol, J. Shin, G. S. Jeon, Y. Skourski, J.-S. Rhyee, and H. Berger, Phys. Rev. B 90, 184402 (2014).

[29] H. Zhang, Z. Zhao, D. Gautreau, M. Raczkowski, A. Saha, V. O. Garlea, H. Cao, T. Hong, H. O. Jeschke, S. D. Mahanti, T. Birol, F. F. Assaad, and X. Ke, Phys. Rev. Lett 125, 037204 (2020).

[30] H. Effenberger, Z. Kristallogr. 175, 61 (1986).

[31] I. Živković, D. M. Djokić, M. Herak, D. Pajić, K. Prša, P. Pattison, D. Dominko, Z. Micković, D. Cinčić, L. Forró, H. Berger, and H. M. Rønnow, Phys. Rev. B 86, 054405 (2012).

[32] T. Cvitanić, V. Šurija, K. Prša, O. Zaharko, I. Kupčić, P. Babkevich, M. Frontzek, M. Požek, H. Berger, A. Magrez, H. M. Rønnow, M. S. Grbić, and I. Živković, Phys. Rev. B 98, 054409 (2018).

[33] S. Lee, W.-J. Lee, J. van Tol, P. L. Kuhns, A. P. Reyes, H. Berger, and K.-Y. Choi, Phys. Rev. B 95, 054405 (2017).

[34] N. Novosel, W. Lafargue-Dit-Hauret, Z. Rapljenović, M. Dragičević, H. Berger, D. Cinčić, X. Rocquefelte, and M. Herak, Phys. Rev. B 99, 014434 (2019).

[35] V. Šurija, K. Prša, H. M. Rønnow, M. Boehm, and I. Živković, 2013, doi:10.5291/ILL-DATA.4-01-1295.

[36] V. Šurija, K. Prša, H. M. Rønnow, P. Steffens, and I. Živković, 2015, doi:10.5291/ILL-DATA.4-01-1459.

[37] See Supplemental Material at http://link.aps.org/supplemental/ 10.1103/PhysRevB.103.L020409 for details.

[38] S. Toth and B. Lake, J. Phys.: Condens. Matter 27, 166002 (2015).

[39] R. A. Cowley, W. J. L. Buyers, P. Martel, and R. W. H. Stevenson, Phys. Rev. Lett. 23, 86 (1969).

[40] T. Huberman, R. Coldea, R. A. Cowley, D. A. Tennant, R. L. Leheny, R. J. Christianson, and C. D. Frost, Phys. Rev. B 72, 014413 (2005).

[41] A. Mezio, C. N. Sposetti, L. O. Manuel, and A. E. Trumper, Europhys. Lett. 94, 47001 (2011).

[42] H. Shao, Y. Q. Qin, S. Capponi, S. Chesi, Z. Y. Meng, and A. W. Sandvik, Phys. Rev. X 7, 041072 (2017).

[43] S.-L. Yu, W. Wang, Z.-Y. Dong, Z.-J. Yao, and J.-X. Li, Phys. Rev. B 98, 134410 (2018).

[44] E. A. Ghioldi, M. G. Gonzalez, S.-S. Zhang, Y. Kamiya, L. O. Manuel, A. E. Trumper, and C. D. Batista, Phys. Rev. B 98, 184403 (2018).

[45] F. Ferrari and F. Becca, Phys. Rev. X 9, 031026 (2019).

Correction: Support information was missing in the Acknowledgment section and has been inserted. 